\title{
EVALUACIÓN DEL PROGRAMA TODOS A APRENDER EN LA ENSEÑANZA DE LENGUAJE Y MATEMÁTICAS EN EL MUNICIPIO DE PUERTO ESCONDIDO-CÓRDOBA
}

\author{
Evaluation of the program Todos a Aprender (Everybody to learn) in language and mathematics \\ teaching at the Puerto Escondido-Córdoba's municipality
}
Avaliação do programa Todos a Aprender no ensino de linguagem e matemáticas no município de Puerto Escondido-Córdoba.

RECIBIDO:17DEMARZODE 2016

\section{RESUMEN}

Con este artículo se pretende presentar los resultados de un estudio tendiente a analizar el impacto del programa Todos a Aprender en la formación pedagógica y la práctica educativa de los docentes de básica primaria de las Instituciones Educativas Oficiales del municipio de Puerto Escondido, en el departamento de Córdoba. El estudio tiene un enfoque cualitativo, con un diseño de estudio de casos, con tres categorías de análisis: concepciones, desarrollo profesional docente y prácticas educativas. Para su desarrollo se tienen en cuenta las siguientes fases: proyección de las comunidades de aprendizaje, ejecución del desarrollo profesional situado y acompañamiento en el aula Los resultados demuestran un efecto positivo sobre la enseñanza e instrucción orientada por los docentes de básica primaria, especialmente en sus metodologías, estrategias didácticas, desarrollo profesional situado y las prácticas evaluativas, así como en el aprendizaje de los estudiantes. Para el alcance de este objetivo se busca consolidar una comunidad de aprendizaje conformada por todos los docentes de educación básica primaria de las 16 instituciones educativas del municipio de Puerto Escondido - Córdoba, donde se realicen procesos de planeación, ejecución, observación y retroalimentación de las clases, por medio de equipos de estudio, teniendo en cuenta las áreas de conocimiento y perfiles de cada uno de los docentes. Esta metodología del estudio de clase busca generar mejoramiento en la calidad educativa de los estudiantes, así como también pretende generar en los docentes motivación hacia su quehacer pedagógico

\section{Palabras Clave: Comunidad de} aprendizaje (CDA), metodología del estudio de clase (MEC), calidad educativa, estudio de casos, formación docente, desarrollo profesional situado (DPS).
EVALUADO:18DE ABRILDE 2016

ACEPTADO:25 DE ENERO DE 2016
Alex Mauricio Díaz (Colombia) alexmau995@gmail.com

Maestría en Educación Sué Caribe Universidad de Córdoba

PARA CITAR ESTE ARTÍ́CULO/TO CITE THIS ARTICLE/PARA CITAR ESTE ARTIGO:

\section{ABSTRACT}

This paper aims to present the results of a study that analyze the impact of the program Everybody to Learn in the pedagogical training and educational practice of elementary teachers of Official Educational Institutions of the municipality of Puerto Escondido in the department of Cordoba. The study has a qualitative approach, with a case study design, and three categories of analysis: conceptions, teacher's professional development, and educational practices. For its development, the following phases are considered: projection of learning communities, execution of situated professional development, and classroom accompaniment. The results show a positive effect on teachings and instructions given by the elementary education teachers, especially in their methodologies, didactic strategies, situated professional development and assessment practices, as well as in student learning. To achieve this goal, we seek to consolidate a learning community comprising all elementary school teachers of 16 educational institutions of the municipality of Puerto Escondido - Córdoba, where planning, implementation, observation and feedback processes are carried out, through study teams that take into account the areas of knowledge and profile of each teacher. The class study methodology aims to improve the quality of education of the students, as well as to motivate teachers towards their pedagogical labor.

KEYWORDS: learning community (CDA in Spanish), class study methodology (MEC in Spanish), educational quality, case study, teacher training, situated professional development (DPS in Spanish).

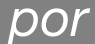

\section{RESUMO}

Com este artigo pretende-se apresentar os resultados de um estudo tendente a analisar o impacto do programa Todos a Aprender na formação pedagógica e a prática educativa dos docentes de básica primária das Instituições Educativas Oficiais do município de Puerto Escondido, no departamento de Córdoba. O estudo tem uma focagem qualitativa, com um desenho de estudo de casos, com três categorias de análise: concepções, desenvolvimento profissional docente e práticas educativas. Para o seu desenvolvimento se têm em conta as seguintes fases: projeção das comunidades de aprendizado, execução do desenvolvimento profissional situado e acompanhamento na aula. Os resultados demostram um efeito positivo sobre o ensino e instrução orientada pelos docentes de básica primária, especialmente nas suas metodologias, estratégias didáticas, desenvolvimento profissional situado e as práticas avaliatórias, assim como no aprendizado dos estudantes.Para o alcance deste objetivo procura-se consolidar uma comunidade de aprendizado conformada por todos os docentes de educação básica primária das 16 instituições educativas do município de Puerto Escondido - Córdoba, onde se realizem processos de planejamento, execução, observação e retroalimentação das aulas, por meio de equipes de estudo, tendo em conta as áreas de conhecimento e perfis de cada um dos docentes. Esta metodologia do estudo de aula procura gerar melhoramento na qualidade educativa dos estudantes, assim como também pretende gerar nos docentes motivação para o seu quefazer pedagógico.

PALAVRAS CHAVE: comunidade de aprendizado (CDA), metodologia do estudo de aula (MEC), qualidade educativa, estudo de casos, formação docente, desenvolvimento profissional situado (DPS).. 


\section{INTRODUCCIÓN}

El propósito de la presente investigación es analizar el impacto de la comunidad de aprendizaje ${ }^{1}$ (CDA) en las prácticas de enseñanza de lenguaje y matemáticas de los docentes de Básica Primaria en el municipio de Puerto Escondido - Córdoba. Esto implica una revisión de los procesos de planeación, ejecución, observación y retroalimentación de las clases, al igual que una caracterización de la metodología del estudio de clase ${ }^{2}$ (MEC), utilizada como estrategia pedagógica en dichas comunidades. Todo esto bajo el programa Todos a Aprender ${ }^{3}$ (PTA) como una propuesta diseñada e implementada por el Ministerio de Educación Nacional de Colombia para mejorar los aprendizajes de los estudiantes de la educación básica primaria en las áreas de lenguaje y matemáticas, especialmente en las instituciones focalizadas por presentar bajos desempeños, de acuerdo con los resultados de las pruebas nacionales de evaluación, pruebas Saber (ICFES, 2010), en las que la función principal es la de: "Mejorar las condiciones de aprendizaje en los establecimientos educativos focalizados y, con ello, el nivel de las competencias básicas de los estudiantes matriculados en ellos entre transición y quinto grado" (MEN, 2012).

La sociedad en la que nos desenvolvemos hoy en día exige una educación en la que nuestros jóvenes sean ciudadanos competentes en un medio cada vez más exigente que evoluciona con criterios de calidad y demanda mayor capacidad de adaptación a las nuevas circunstancias. Esto implica que en la escuela se formulen estrategias pedagógicas que orienten los procesos de enseñanza hacia el desarrollo de capacidades, destrezas y actitudes en nuestros educandos, para que, de este modo, en ellos se fortalezca una serie de competencias que les permitan desempeñarse de manera exitosa en los retos que se les presenten a lo largo de su vida (OCDE, 1995).

Se habla, entonces, de una educación que asegure mínimamente en los niños y jóvenes la adquisición de los conocimientos, capacidades, destrezas y actitudes necesarias para desempeñarse en la vida adulta. De esta

\footnotetext{
1 En lo sucesivo del texto se utilizará la abreviatura $\mathrm{CDA}$ para hacer referencia a las comunidades de aprendizaje.

2 En lo sucesivo del texto se utilizará la abreviatura MEC para hacer referencia a las metodologías del estudio de clase.

3 En lo sucesivo del texto se utilizará la abreviatura PTA para hacer referencia al programa Todos a Aprender.
}

manera, el mejoramiento de la calidad y el aprendizaje ha de tener un papel más decisivo en el marco del desarrollo mundial en los próximos años. Por eso, el diseño de estrategias es un factor clave si se busca mejorar las posibilidades de educación de millones de niños que son incapaces de leer y escribir o no disponen de competencias aritméticas básicas ${ }^{4}$.

En tal sentido, se presenta una crisis del aprendizaje en nuestros estudiantes, de la que resulta necesario ocuparse si se quiere formar en competencias en áreas como el lenguaje y matemáticas, puesto que estas son necesarias para conseguir un trabajo digno que le permita la realización y el desarrollo a plenitud de su proyecto de vida. Por esta razón, se necesitan docentes capacitados, con dedicación suficiente, que sepan descubrir y prestar atención especial a quienes tienen dificultades de aprendizaje, y que estén apoyados, a su vez, por sistemas educativos bien administrados, de modo que puedan liberar su potencial y contribuir al mejoramiento de la calidad de los aprendizajes de sus estudiantes. Ahora bien, si los docentes son un factor clave para la calidad de la educación, se debe considerar que, al mejorar su acción educativa, se puede conseguir al mismo tiempo mayores niveles de calidad en el funcionamiento de las escuelas (Marchesi, 2011). Esto sugiere que la calidad de la educación mejora cuando se apoya a los docentes y cuando las propuestas para mejorar su situación se basan en enfoques contextuales e integrales, en los que se tengan en cuenta todos los factores que contribuyen a facilitar el trabajo de los profesores, puesto que ellos no pueden cargar con toda la responsabilidad de los problemas asociados a la calidad de la educación.

Otro elemento de gran importancia, cuando se habla de calidad educativa, es la evaluación. Las pruebas internacionales y regionales que se han implementado en muchos países en estos últimos años, brindan una buena base para llevar a cabo el seguimiento de esos progresos. Por otro lado, debido a que la meta de muchos países es que para el 2030 todos los niños y los jóvenes adquieran competencias básicas en lectura, escritura y matemáticas, es necesario direccionar el seguimiento de los progresos a fin de asegurar que los países avancen hacia el logro de esa meta (UNESCO, 2014). Esto demanda

\footnotetext{
4 La UNESCO (2014), publicó el undécimo Informe de seguimiento de la Educación para Todos (EPT). Aquí se muestran los avances logrados por los diferentes países en la consecución de los objetivos mundiales de educación acordados en el año 2000. También se presentan argumentos convincentes en favor de que la educación ocupe un lugar central en la agenda mundial para el desarrollo después de 2015.
}

Evaluación del programa Todos a Aprender en la enseñanza de lenguaje y matemáticas en el municipio de Puerto EscondidoCórdoba
I Panorama I pp. 46-60 I Volumen 10 I Número 19 I Julio-diciembre I 2016 
Alex Mauricio

Díaz I

Panorama |

pp. 46-60।

Volumen 10 I

Número 19।

Julio-diciembre

2016 |

(1)$$
\text { }
$$

Nacional, secretarías de educación y en general todos los colombianos; todo esto con el fin para contrarrestar los efectos de esos factores propios de entornos familiares, escolares o comunales poco favorables que influyen negativamente en el aprendizaje de los estudiantes.

Se hace necesario evaluar el impacto que ha tenido este programa, particularmente en las zonas rurales, donde los resultados en las áreas evaluadas en las pruebas estandarizadas muestran una gran parte de la población de estudiantes del país en niveles de desempeño bajo $o$ insuficiente en las diferentes pruebas aplicadas. Además, puesto que una de las estrategias que propone el PTA es la transformación de las practicas pedagógicas de los docentes, es pertinente conocer sus percepciones y concepciones al respecto de cómo este programa ha tenido influencia en los diversos factores involucrados en su quehacer, en la construcción, el diseño y la puesta en práctica de propuestas pedagógicas en el aula.

Por esta razón, el propósito de la presente investigación es analizar el impacto del PTA en la formación pedagógica y la práctica educativa de los docentes de básica primaria de las instituciones educativas oficiales, ubicadas en la zona rural del municipio de Puerto Escondido en el departamento de Córdoba. Este trabajo implica una revisión de los procesos de planeación, ejecución, observación y realimentación de las clases, al igual que una caracterización de la metodología del estudio de clase (MEC), utilizada como estrategia pedagógica en dichas comunidades. Además, es importante identificar aquellos factores asociados al desarrollo profesional situado (DPS), que afectan la calidad de los aprendizajes de los estudiantes, en donde la capacidad de los docentes para enseñar es el más crítico de todos (Mckinsey, 2007). Esta capacidad se expresa en el conocimiento que tienen los docentes sobre qué y cómo aprenden sus estudiantes (Bransford, Brown y Cocking, 2000); así mismo, el dominio sobre una disciplina y las prácticas más adecuadas para enseñar dicha disciplina (Celis, Duque y Díaz, 2013). Es de gran relevancia abarcar estos aspectos, puesto que la profesionalización de la enseñanza es reconocida como el factor determinante para mejorar la capacidad de los aprendizajes.

En este contexto, el trabajo del PTA en las instituciones se configura a partir de comunidades de aprendizaje (CDA), entendidas como "un proyecto de transformación social y cultural de un centro educativo y de su entorno, para conseguir una sociedad de la información 
para todas las personas" (Valls, 2005). Por eso, es necesario conocer la estrategia de trabajo de los docentes que conforman una CDA en las instituciones educativas oficiales, con el propósito de identificar las prácticas pedagógicas para mejorar los aprendizajes de los estudiantes y despertar la motivación por aprender. De otra parte, la enseñanza del lenguaje y las matemáticas en la educación básica primaria son los ejes fundamentales y pilares del proceso de la educación, ya que la adquisición de estas competencias garantiza la calidad educativa y permite que los educandos desarrollen la capacidad humana y potencial de comprender, analizar y reflexionar sobre las situaciones que se les presentan a diario. Sin embargo, para valorar a plenitud este proceso de calidad educativa y de aprendizaje significativo, se hace necesario conocer los resultados que han obtenido los estudiantes, a partir de la implementación del PTA en la institución, para determinar si resulta significativo poner en marcha estas estrategias y si en realidad el impacto que se genera en los aprendizajes de los estudiantes está asociado a su puesta en práctica en las aulas de clase.

En este orden de ideas, se muestran los resultados de las pruebas Pisa año 2009 en las áreas de lenguaje y matemáticas.

Tabla 1 - Cómo hicieron los sistemas educativos con mejor desempeño del mundo para alcanzar sus objetivos.
Visto esto, se puede decir que las metodologías de estudio de clase (MEC), el trabajo en equipo y la formación pedagógica mediante las $\mathrm{CDA}$ de docentes han impactado significativamente en el mejoramiento de la calidad educativa de los sistemas educativos que vienen implementado estas estrategias metodológicas para el proceso de la enseñanza y de la instrucción a partir del trabajo cooperativo y colaborativo desde hace varias décadas.

\section{Metodología}

El enfoque que posibilita aproximarse a la naturaleza humana y a la concepción de las personas, desde un pensamiento crítico y la vivencia diaria del contexto donde se asientan tales comunidades es el enfoque cualitativo. Por tal razón, la investigación cualitativa focaliza aspectos de tipo vivencial, además del contexto socio cultural, el entorno, las costumbres y concepciones. El enfoque cualitativo permite conceptualizar sobre la realidad con base en el comportamiento, los conocimientos, las actitudes y los valores que guían la conducta de las personas estudiadas, en este caso, los docentes del sector oficial. El proceso de investigación cualitativo explora de manera sistemática los conocimientos y valores que comparten los individuos en un determinado contexto espacial y temporal (Bonilla, 2005).
Evaluación del programa Todos a Aprender en la enseñanza de lenguaje y matemáticas en el municipio de Puerto Escondido-

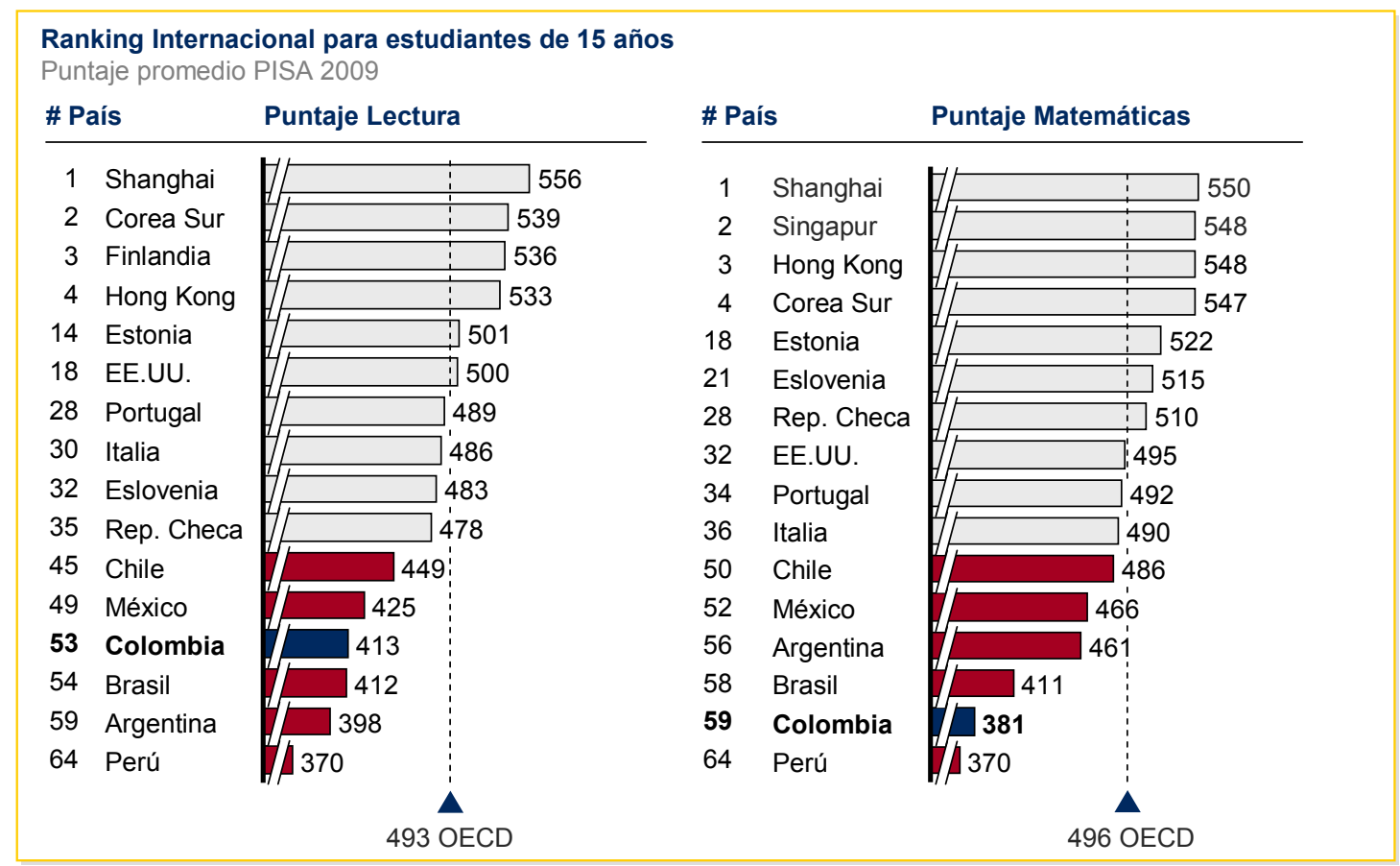

Fuente: estudio de Mckinsey y Company (2012). www.oecd.org

I Panorama I pp. $46-60$ I Volumen 10 I Número 19 I Julio-diciembre I 2016 
Alex Mauricio

Díaz I

Panorama I

pp. 46-60 I

Volumen 101

Número 19|

Julio-diciembre

2016 |

\section{,}

Este proyecto de investigación se posiciona dentro del enfoque cualitativo, desde donde se propone contribuir a la comprensión y análisis de la formación pedagógica en los procesos de enseñanza del lenguaje y las matemáticas de los docentes que laboran en las instituciones educativas rurales del municipio de Puerto Escondido que han sido focalizadas por el proyecto de investigación. De igual forma, se busca indagar acerca de las percepciones que tienen los docentes sobre su práctica pedagógica y su incidencia en el aprendizaje de los estudiantes. El enfoque de investigación cualitativa permite utilizar diversos instrumentos para comprender la vida social por medio de significados y desde una perspectiva holística, pues se trata de entender el conjunto de cualidades interrelacionadas que caracterizan a un determinado fenómeno (Taylor y Bodgan, 1987; Mejía, 2004). Los datos recolectados en el desarrollo de esta investigación, son de orden descriptivo y permitirán conocer la voz, las concepciones y las conductas observables de los sujetos involucrados en el estudio.

Una investigación que se enmarca en el enfoque cualitativo es abierta, flexible, y se construye durante el trabajo de campo o a partir de las fases que implican la realización del estudio. De manera que este enfoque permite aproximarse a la naturaleza humana y a la concepción de las personas, desde un pensamiento crítico y sus vivencias para conceptualizar sobre la realidad con base en el comportamiento, los conocimientos, las actitudes y los valores que guían la conducta de las personas estudiadas. Al respecto, Hernández, Fernández y Baptista (2010), señalan que existen varias realidades subjetivas construidas en la investigación, las cuales varían en su forma y contenido entre individuos, grupos y culturas, por lo que el enfoque cualitativo permite describir, comprender e interpretar los fenómenos, por medio de las percepciones y significados producidos por las experiencias de los participantes. Desde esta perspectiva, se han obtenido suficientes datos que se han analizado y que han permitido comprender lo que los docentes afirman con relación a su práctica pedagógica y cómo su quehacer se ha visto transformado a partir de la implementación del programa PTA en la institución; del mismo modo, decir, comprender y explorar de manera sistemática los conocimientos y valores que comparten en su ámbito de trabajo: el aula de clases.

\section{TIPO DE ESTUDIO}

El trabajo de investigación sigue un modelo analítico basado en casos, en donde se utiliza una metodología interpretativa que busca conocer y comprender en profundidad los casos que son objeto de análisis, los cuales se consideran complejos y que están en constante funcionamiento (Stake, 1999). Por ello, este tipo de investigaciones se caracterizan por poseer un análisis detallado, comprensivo y minucioso del caso que manifiesta el objeto del estudio. En tal sentido, es un trabajo particularista, descriptivo, heurístico, inductivo y cooperativo en términos de la relación de los actores involucrados y el investigador. Los estudios de casos poseen una gran riqueza metodológica que los hace importantes en diversos paradigmas de investigación. Stake (2006) y Hammersley (2007), reconocen que es complejo y problemático intentar asociar el estudio de caso con una forma específica de investigación, debido a que se ha utilizado tanto en el enfoque experimental como en otras aproximaciones cuantitativas y en la investigación cualitativa. Desde esta perspectiva, el estudio de caso no está definido por un método específico, sino por su objeto de análisis. Otra característica del estudio de casos es el de la particularización, y no de la generalización.

A partir del abordaje metodológico de un caso específico, se procura conocerlo bien en su totalidad. Al ser cada caso único, implica el conocimiento de los otros casos, pero la finalidad primera es la comprensión de este último (Stake, 1999). Las condiciones a partir de las cuales se delimita qué puede ser el caso a estudiar también están marcadas por poder establecer adecuadamente los límites con su entorno. Mertens (2005) citado por Hernández et al. (2010), se refiere al estudio de caso como una investigación sobre un individuo, grupo, organización, comunidad o sociedad, que es visto y analizado como una entidad. Igualmente, se debe considerar el contexto en el cual se desenvuelve el sujeto a estudiar. Por esta razón, se sustenta que el presente trabajo se clasifica como un estudio de caso, ya que es importante considerar todos los aspectos y las condiciones en que se desempeñan los docentes que son considerados los casos objeto de estudio, lo que obliga a hacer las indagaciones empíricas que investigan determinado fenómeno dentro de su contexto en la vida real (Yin, 2009 citado por Hernández et al., 2010). En este caso, el fenómeno que se busca analizar es el impacto 
del programa PTA en la formación pedagógica y la práctica educativa de los docentes de básica primaria de las instituciones educativas focalizadas en el municipio de Puerto Escondido.

\section{POBLACIÓN PARTICIPANTE}

Para este estudio fueron seleccionados dos (2) de los diez (10) establecimientos educativos rurales de educación básica primaria del municipio de Puerto Escondido Córdoba, que componen la totalidad de las instituciones educativas que han participado en cada una de las fases de la implementación del PTA en este municipio y que cumplen con algunos criterios para la selección de los casos (Taylor y Bogdan, 1987), teniendo en cuenta las consideraciones de Stake (1999), para determinar aquellas características que deben cumplir los participantes, y que permitan delimitar a la población o participantes del estudio. Atendiendo a esto, se parte inicialmente de la disposición y voluntariedad que tenían los docentes que laboran en las Instituciones educativas seleccionadas, para participar en la investigación y aportar toda la información necesaria que se requiriera por parte de la unidad investigativa.

\section{CATEGORÍAS DE ANÁLISIS}

En esta investigación se han definido las siguientes categorías, en relación con los referentes conceptuales $y$ teniendo en cuenta el rastreo documental que se ha llevado a cabo por parte del investigador, con el fin de dar soporte a las intenciones que se tiene en el marco de este trabajo. En la siguiente tabla se detallan las categorías, sus dimensiones y la asociación que tienen con los objetivos de la investigación.

Tabla 2

\begin{tabular}{|c|c|c|}
\hline $\begin{array}{l}\text { OBJETIVOS } \\
\text { ESPECÍFICOS }\end{array}$ & DIMENSIONES & CATEGORIAS \\
\hline $\begin{array}{l}\text { Describir las } \\
\text { percepciones } \\
\text { que tienen } \\
\text { los docentes } \\
\text { acerca de la } \\
\text { implementación } \\
\text { de las } \\
\text { comunidades de } \\
\text { aprendizaje en } \\
\text { las instituciones } \\
\text { educativas. }\end{array}$ & $\begin{array}{l}\text { Contexto de la } \\
\text { escuela. } \\
\text { Interacción } \\
\text { docente - tutor. } \\
\text { Comportamientos } \\
\text { y actitudes. }\end{array}$ & CONCEPCIONES \\
\hline
\end{tabular}

\begin{tabular}{|c|c|c|}
\hline $\begin{array}{l}\text { OBJETIVOS } \\
\text { ESPECÍFICOS }\end{array}$ & DIMENSIONES & CATEGORÍAS \\
\hline $\begin{array}{l}\text { Analizar el } \\
\text { desarrollo de } \\
\text { la formación } \\
\text { pedagógica en } \\
\text { los procesos de } \\
\text { enseñanza del } \\
\text { lenguaje y las } \\
\text { matemáticas } \\
\text { mediante la } \\
\text { implementación } \\
\text { de las } \\
\text { comunidades de } \\
\text { aprendizaje. } \\
\text { Valorar la } \\
\text { práctica educativa } \\
\text { por medio de } \\
\text { los resultados } \\
\text { obtenidos en } \\
\text { la evaluación } \\
\text { externa pruebas } \\
\text { Saber Tercero } \\
\text { y Quinto ciclo } \\
\text { 2017 - 2018 en } \\
\text { las instituciones } \\
\text { educativas rurales } \\
\text { focalizadas en } \\
\text { el municipio } \\
\text { de Puerto } \\
\text { Escondido. }\end{array}$ & $\begin{array}{l}\text { Conocimiento } \\
\text { didáctico de los } \\
\text { contenidos. } \\
\text { Desarrollo } \\
\text { profesional } \\
\text { situado. } \\
\text { Formación } \\
\text { pedagógica. } \\
\text { Pares académicos. }\end{array}$ & $\begin{array}{l}\text { DESARROLLO } \\
\text { PROFESIONAL } \\
\text { DOCENTE }\end{array}$ \\
\hline
\end{tabular}

Fuente: Elaboración propia del autor

El análisis de los datos en esta investigación se basa en lo propuesto por Strauss y Corbin (2002), donde a través de un proceso analítico se identifican los conceptos y se descubren en los datos sus propiedades y dimensiones, así como también se relacionan las categorías a sus subcategorías teniendo en cuenta sus propiedades y dimensiones. Las codificaciones fueron hechas a partir de la anterior matriz de análisis, utilizando el software para análisis Atlas Ti, versión 7.

\section{TÉCNICAS DE RECOLECCIÓN DE INFORMACIÓN}

Las fuentes de información permiten visualizar aspectos valiosos y heterogéneos en la búsqueda de la información, propiciando una participación activa y dinámica por medio de la lectura, la observación, el análisis, la interpretación y, por supuesto, la argumentación. En el campo de la investigación, proporcionan complementariamente datos empíricos de la realidad compleja estudiada, basados en criterios de credibilidad y validez. En este sentido, la credibilidad de un estudio cualitativo se relaciona con el uso que se haya hecho de un conjunto de recursos técnicos (Valles, 1997). Las técnicas e instrumentos que permitieron la consolidación
| Evaluación del programa Todos a Aprender en la enseñanza de lenguaje y matemáticas en el municipio de Puerto EscondidoCórdoba
I Panorama I pp. 46-60 I Volumen 10 I Número 19 I Julio-diciembre | 2016 
y elaboración del presente informe de investigación se definen a continuación.

Alex Mauricio

Díaz |

La observación participante es el proceso que faculta a los investigadores a aprender acerca de las actividades de las personas en estudio en un escenario natural mediante la observación y participando en sus actividades (DeWalt y DeWalt, 2002). Este proceso de aprendizaje se propicia a través de la exposición y el involucrarse en las actividades de rutina de los participantes en el escenario del investigador (Schensul, Schensul y LeCompte,1999). Desde esta perspectiva, la observación participante permite establecer relaciones con una comunidad y aprender a actuar al punto de mezclarse con ella y hacer que sus miembros actúen de forma natural. Posteriormente, el investigador se aleja del escenario para sumergirse en los datos para comprender y ser capaz de escribir acerca de lo que observa. La observación participante depende del registro de notas de campo, las cuales deben ser completas, precisas y detalladas, no deben incluir solo descripciones de lo que ocurre, sino también un registro de los sentimientos e interpretaciones (Taylor y Bodgan, 1987). La observación participante puede ser usada para ayudar a responder preguntas de investigación, construir teoría, o para generar o probar hipótesis (Dewalt y Dewalt, 2002). Es considerada como una forma de incrementar la validez, ya que las observaciones pueden ayudar al investigador a tener una mejor comprensión del contexto y el fenómeno en estudio.

De modo que la observación participante se constituye en esta investigación como la más substancial de las estrategias de producción de la información, pues la meta fundamental es la reflexión de la acción didáctica de los maestros dentro del aula, por medio de la vivencia de las

Panorama I pp. 46-60। Volumen 10 I Número 19। Julio-diciembre 2016 | propuestas por este mismo programa para la enseñanza del lenguaje y las matemáticas en las instituciones educativas, esta información será complementada, precisada y reevaluada con los datos obtenidos mediante la observación de la clase aplicada en la fase de ejecución. Dicha

52 | observación posibilita el estudio de casos de la práctica de los docentes de la educación básica primaria en las instituciones educativas seleccionadas.

\section{Entrevistas}

La entrevista es una técnica de investigación cualitativa, cuyo objetivo es la recolección de datos a partir de las propias palabras, pensamientos y reflexiones del sujeto entrevistado. Autores como Benney y Hughes (1970), afirman que la entrevista es una herramienta para excavar, puesto que a partir de relatos verbales es posible adquirir conocimientos y adentrarse sobre la vida social del otro. Dentro de los distintos tipos de entrevistas en investigación cualitativa se distinguen: entrevista estructurada, semiestructurada y en profundidad. Es de interés en este estudio referirse a las entrevistas cualitativas semiestructuradas, puesto que los encuentros cara a cara entre el investigador y los informantes se orientan hacia la comprensión de las perspectivas que tienen los docentes respecto de sus experiencias en el aula. Las entrevistas semiestructuradas siguen el modelo de una conversación entre iguales, y no de un intercambio formal de preguntas y respuestas. Permiten estudiar un número relativamente grande de personas en un lapso relativamente breve, si se le compara con el tiempo que requeriría otro tipo de investigación cualitativa (Taylor y Bogdan, 1987). Además, brindan un mayor grado de libertad y autonomía al entrevistado que el que proporciona una entrevista estructurada y un menor grado que la entrevista en profundidad.

En el aspecto metodológico, las entrevistas semiestructuradas implican que la situación y los estímulos sean iguales, así como la redacción de las preguntas debe proveer el mismo significado para todos; además, es necesario que el orden de las preguntas sea el mismo para todos los entrevistados, con el fin de lograr un contexto de equivalencias. En definitiva, la entrevista semiestructurada pretende comprender más que explicar, busca maximizar el significado, dar con la respuesta subjetivamente sincera (Ruiz, 2010).

En esta investigación, la entrevista va a permitir la construcción de una reflexión de cómo el docente desarrolla sus clases, los referentes educativos y materiales que utiliza, las experiencias significativas que construye con sus estudiantes y la transformación que ha logrado en sus aprendizajes. 
TRLANGULACIÓN

La triangulación es un tipo de control de calidad que debiera ser aplicada a todo tipo de investigación cualitativa. Algunos autores (Stake, 1999; Ruiz, 1999), afirman que existen distintos tipos de triangulación. Según Hernández et al. (2010), la triangulación de datos se emplea para hacer un análisis de los mismos utilizando diferentes técnicas y desde diversos ángulos.

En el marco de esta investigación se utiliza la triangulación, en la que se utiliza una variedad de datos de diversas fuentes. En este caso se va a realizar una triangulación entre la entrevista semiestructurada, el instrumento de observación y los resultados de las pruebas Saber aplicadas en las instituciones educativas focalizadas. Cada una de las técnicas busca recolectar datos específicos a través de distintas fuentes de información, sobre aspectos del objeto de estudio definido desde la complejidad, abordaje que fue realizado en concordancia con el marco teórico de la investigación.

\section{FASES DE LA INVESTIGACIÓN}

FASE DE PROYECCIÓN DE LA COMUNIDAD DE APRENDIZAJE

Esta fase del desarrollo de esta propuesta comprendió la conformación de la CDA, a partir del análisis y estudio de las metas propuestas. Estos objetivos están encaminados a mejorar las condiciones de aprendizaje para los estudiantes matriculados entre transición y quinto grado de básica primaria, y lograr que por lo menos el $25 \%$ de los estudiantes asciendan del nivel insuficiente, al menos en las áreas de lenguaje y matemáticas de la prueba Saber $3^{\circ}$ y $5^{\circ}$ que será aplicada en 2017 y 2018, y cuyos resultados serían publicados en marzo de 2018 - 2019, lo cual va a ser acordado en los establecimientos educativos oficiales rurales seleccionados, mediante actas de sensibilización, acuerdos de gestión y socialización con toda la comunidad educativa de las instituciones focalizadas.

\section{FASE DE EJECUCIÓN DEL DESARROLLO PROFESIONAL} SITUADO

A partir de la conformación de la CDA, los docentes de las instituciones focalizadas en compañía del investigador (en su rol de docente tutor), se reunieron mensualmente con el fin de poder recibir orientaciones en torno a las estrategias y a las secuencias didácticas que se deben trabajar en las áreas de lenguaje y matemáticas. Para ello, se proponen las sesiones de trabajo situado (STS), que son los espacios de interacción, integración, planeación y modelamientos de las secuencias didácticas que debe aplicar el grupo de docentes, para luego ser replicada dentro del aula de clases con los materiales y recursos provistos por el PTA. En todo momento del proceso se cuenta con la observación del equipo completo de docentes de la básica primaria, incluidos los casos seleccionados en esta investigación.

\section{FASE DE ACOMPAÑAMIENTO EN EL AULA}

A partir de la planeación que se realiza por grados, se procedió a observar el desarrollo de las clases planeadas por todo el equipo de trabajo, con los instrumentos y protocolos diseñados para la observación de las clases, focalizando aspectos de instrucción, ambiente escolar, gestión del aula y conocimiento didáctico de los contenidos. Luego de este proceso de observación, el tutor se reúne con la CDA para socializar los resultados obtenidos, y entre todos los participantes hacer correcciones, evaluar el proceso de instrucción y dar la retroalimentación final para luego pasar al proceso inicial de planeación de secuencias didácticas, con mayor énfasis en los problemas de aula observados anteriormente. En lo que respecta al aprendizaje de los estudiantes a partir de las reflexiones dentro de la CDA, se establecieron prioridades con relación a lo que los estudiantes deben aprender. La gestión académica se fortalece, ya que se intercambian estrategias aplicadas y se buscan soluciones a problemas comunes e individuales relacionados con la práctica pedagógica. Finalmente, este tipo de encuentros permitió la consolidación de la comunidad de aprendizaje de docentes de las instituciones educativas focalizadas, destacando la importancia del desarrollo profesional situado, el conocimiento didáctico de los contenidos y las estrategias didácticas para la atención de las necesidades educativas de los estudiantes y el mejoramiento de la calidad de la enseñanza de los docentes.

\section{RESULTADOS DE LA INVESTIGACIÓN}

De acuerdo con la situación expuesta a partir de la revisión documental y en función del objetivo que orientó
Evaluación del

programa Todos

a Aprender en

la enseñanza

de lenguaje y

matemáticas

en el municipio

de Puerto

Escondido-

Córdoba
I Panorama

I pp. 46-60

I Volumen 10

I Número 19

I Julio-diciembre

| 2016 
Alex Mauricio

Díaz I este proyecto investigativo en las instituciones educativas oficiales del municipio de Puerto Escondido, focalizadas por el PTA, es decir, la identificación del grado de pertinencia del PTA en la región de Puerto Escondido - Córdoba y el impacto que este programa ha generado en la comunidad educativa de este municipio tomando como base de acción docentes, directivos docentes, estudiantes y padres de familia. Se tienen los siguientes resultados:

\section{COMPONENTE PEDAGÓGICO}

El componente pedagógico se encuentra relacionado con los referentes curriculares nacionales, tales como lineamientos curriculares y estándares elementales de competencias básicas para las áreas de lenguaje y matemáticas. La evaluación de aprendizajes que se presentan en las instituciones educativas del sector oficial de Colombia, las cuales son evaluaciones nacionales como pruebas Saber $3^{\circ}, 5^{\circ}$ y $9^{\circ}$ que evalúa ciencias, matemáticas y lenguaje, y evaluaciones internacionales como pruebas Pisa que evalúa el aprendizaje del lenguaje y matemáticas en estudiantes de educación básica; por último, el uso pertinente de materiales educativos para la enseñanza del área de lenguaje y matemáticas.

Tabla 3

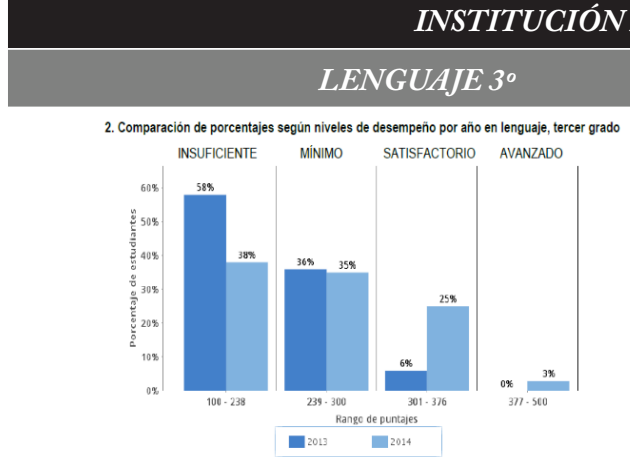

En lo que respecta a la evaluación, de acuerdo con Díaz et al.:

Si atendemos a factores de corte cuantitativo, vemos que tanto las pruebas estandarizadas internacionales (Pisa, 2009; Serce, 2006), como los resultados reportados en las pruebas nacionales (Saber, 2009), evidencian que el nivel de desarrollo de las competencias de los estudiantes colombianos ocupa los niveles más bajos y, por tanto, no logra ser garante ni de desempeños básicos ante las exigencias de este siglo, ni de aprendizajes posteriores (2015).

Por este motivo, el PTA toma como sustento los resultados de las pruebas Pisa y Saber año 2009, de tal modo que se pueda intervenir el currículo de las instituciones educativas oficiales del país ubicadas en sectores rurales de difícil acceso, como son las instituciones educativas focalizadas en esta investigación, demostrando así que resulta pertinente una intervención para mejorar los resultados en las evaluaciones internas y externas.

Finalmente, en las evaluaciones de pruebas Saber $3^{\circ} \mathrm{y}$ $5^{\circ}$ se identifica cómo los resultados comparativos de los años 2013 y 2014, en las áreas de lenguaje y matemáticas, demuestran avances significativos en los procesos de enseñanza y en los niveles de desempeño de los estudiantes.
Panorama I

pp. 46-60 I

Volumen 101

Número 19 |

Julio-diciembre

2016 ।

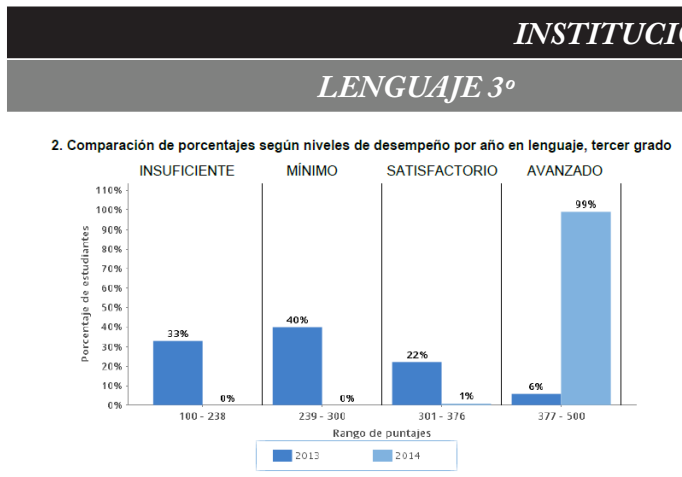

MATEMÁTICAS $3^{\circ}$

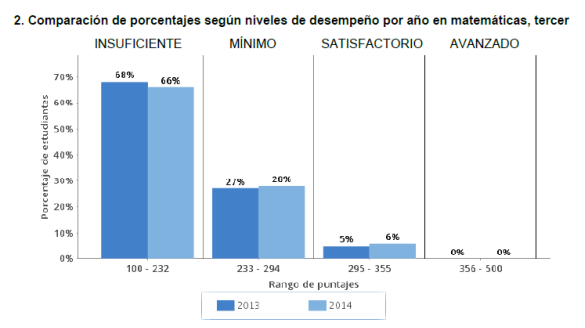




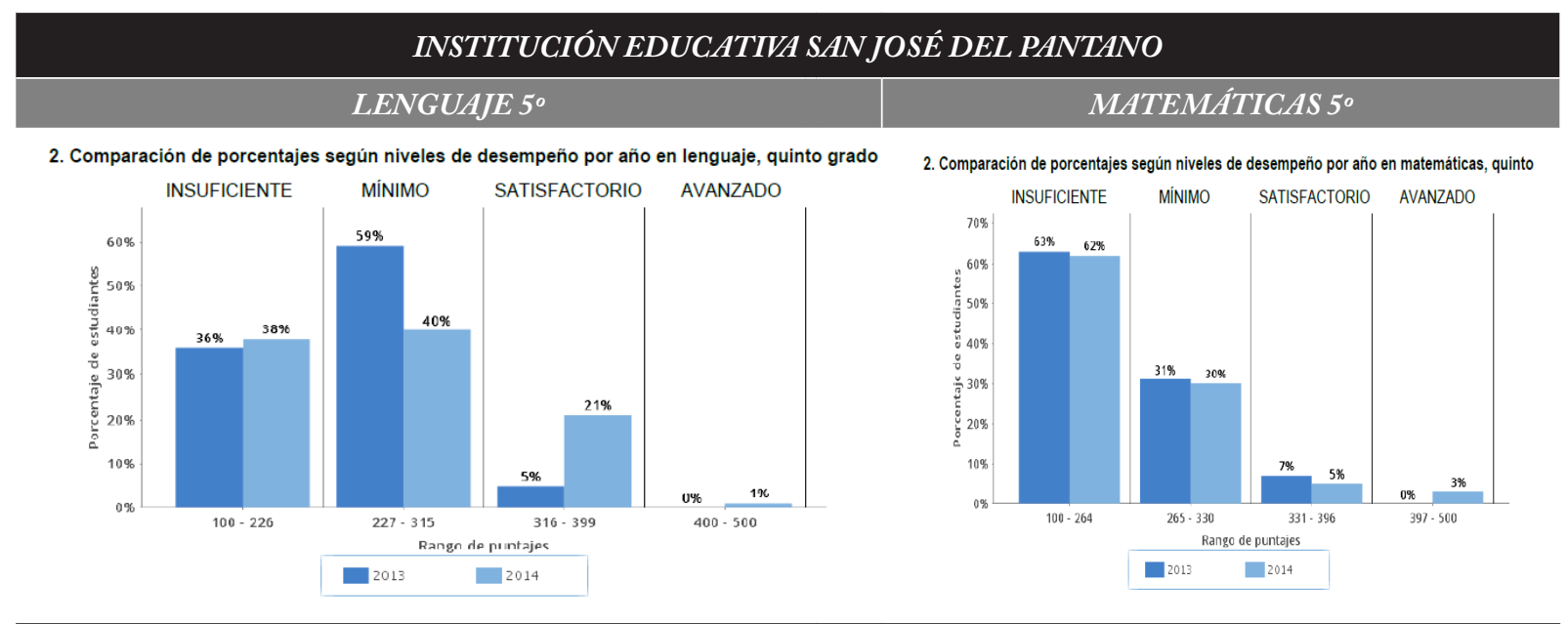

\section{INSTITUCIÓN EDUCATIVA SANJOSÉ DEL PANTANO}

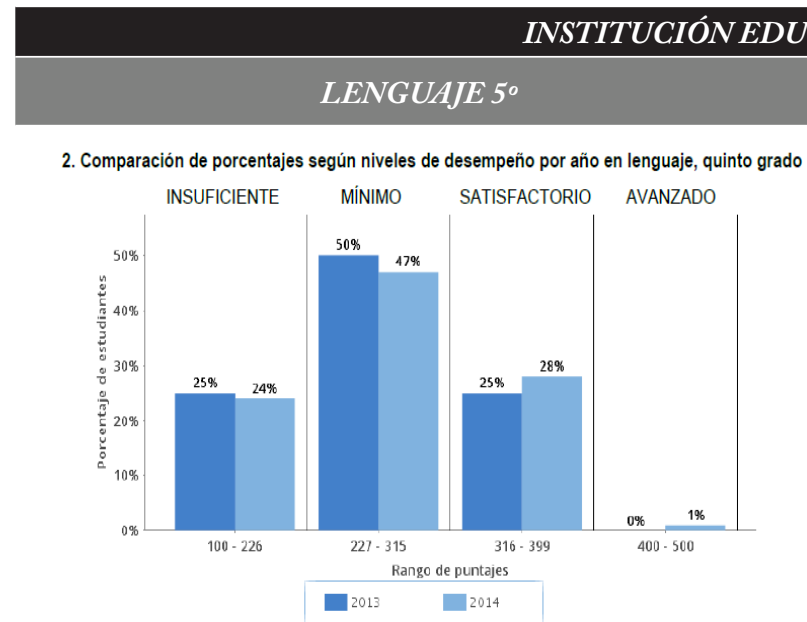

MATEMÁTICAS 5०

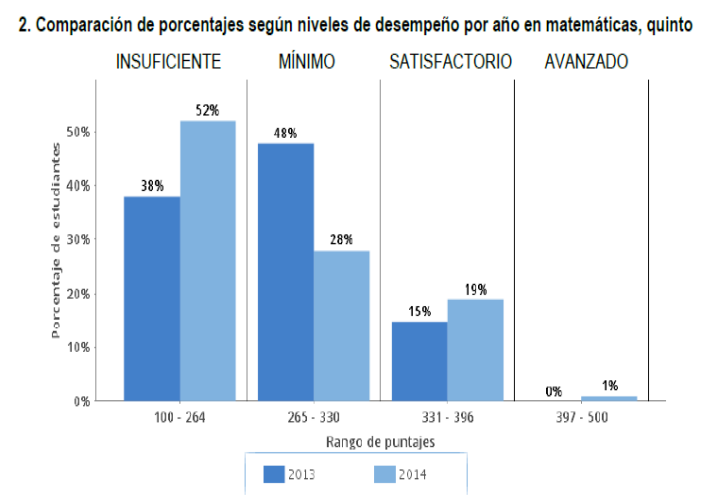

Fuente: Elaboración propia del autor

Al comparar los resultados de la muestra representativa de las instituciones educativas focalizadas en el municipio de Puerto Escondido de la prueba nacional ICFES Saber $3^{\circ}$ y $5^{\circ}$ año 2013 - 2014, se evidencia una mejora significativa en las áreas de lenguaje y matemáticas, ya que se reduce el porcentaje de estudiantes con desempeño insuficiente. Así mismo, aumenta el porcentaje de estudiantes con desempeño mínimo y ubica un porcentaje significativo del total de estudiantes en desempeño satisfactorio, resultado que es coherente con las metas que persigue el PTA en las instituciones educativas focalizadas en todo el país, lo cual evidencia el mejoramiento de los procesos de instrucción y de enseñanza en los docentes en las áreas evaluadas y la consecución de las metas establecidas por el PTA para el municipio de Puerto Escondido en los acuerdos de gestión firmado por la gobernación de Córdoba en representación del secretario de educación departamental, el alcalde del municipio de Puerto Escondido, docentes y directivos docentes de las instituciones educativas focalizadas en la iniciación del PTA desde el año 2013.

\section{COMPONENTE DE CONDICIONES BÁSICAS}

El uso de los materiales educativos (Colección semilla, Nivelemos y Escuela Nueva), en las instituciones educativas focalizadas, ha permitido al docente contar con un material de alta calidad para el desarrollo de las clases y mejorar su proceso de instrucción, pues el estudiante cuenta con un material físico y de fácil acceso para ejercitar su proceso de aprendizaje y, como situación inicial, se halló que, en torno a los contenidos educativos, faltaba pertinencia en los planes de estudio: no se partía de un diagnóstico básico para determinar los contenidos educativos adecuados para cada ciclo de grado, ni se revisaban a fondo los referentes de calidad en la planeación de los mismos. La educación era tradicionalista, a pesar de lo que promulgaban en sus respectivos PEI. La educación matemática se orientaba casi exclusivamente a desarrollar el pensamiento numérico y el proceso de ejercitación, y la mayoría de docentes desconocían los otros pensamientos y procesos (Márquez, 2015). Se dio así un giro total al proceso de instrucción en las instituciones educativas focalizadas en este proyecto
I Panorama I pp. $46-60$ I Volumen 10 I Número 19 I Julio-diciembre | 2016 
investigativo, centrando el proceso en el aprendizaje del estudiante y sus habilidades motrices.

Alex Mauricio

Díaz I

Durante la ejecución del PTA en el municipio de Puerto Escondido, se ha podido certificar que el estudiante esté inmerso en los ambientes de aprendizaje, garantizando a los estudiantes la ruta y el transporte escolar para las instituciones educativas del municipio, mantenimiento y construcción de espacios funcionales para que los estudiantes puedan desarrollar las actividades académicas y deportivas, programa de alimentación escolar y nutrición, y finalmente, apertura de las puertas de las instituciones educativas a las familias y a las diferentes entidades gubernamentales para que puedan garantizar la asistencia escolar cotidiana y el desempeño de las actividades curriculares académicas.

\section{COMPONENTE DE FORMACIÓN SITUADA}

La necesidad de formación y actualización pedagógica del docente es el principio por el cual se da la creación del PTA, dado que desde este programa educativo se pretende darles a los docentes una formación in situ con pertinencia y de gran calidad, atendiendo a la actualización pedagógica y las nuevas tendencias educativas que impactan la instrucción del docente y el aprendizaje del estudiante significativamente, basados en las orientaciones pedagógicas del Ministerio de Educación Nacional para pares académicos, formadores y tutores del PTA, entre otros. Recordemos que:

Las necesidades que dan lugar a programas pueden ser detectadas en las personas o en los grupos para los que se diseñan los programas, pero, también, pueden responder a carencias más o menos generales de la sociedad que, para bien, deberían ser atendidas mediante la formación y capacitación de las personas (Pérez, 2014, p. 169).

Panorama I

Potenciar las prácticas de aula del docente es el objetivo principal del PTA, por lo tanto: "su objetivo está relacionado con la creación, puesta en marcha y mejoramiento de ambientes de aprendizaje efectivos en contextos especialmente difíciles" (MEN, 2013). Para la consecución de este objetivo principal se han desarrollado diferentes estrategias, tales como la consolidación de las comunidades de aprendizaje de las instituciones educativas focalizadas por el PTA y acompañamiento en aula para reflexionar acerca de las prácticas educativas en CDA, lo cual se constituye como soporte a la capacidad de formación y a la cualificación permanente, lo que además se articula con el sistema de evaluación de desempeño a docentes regidos por el decreto ley 1278 de 2002, emanado por el MEN, en el que el docente debe dar cuenta de un conjunto de conocimientos de orden disciplinar y didáctico.

\section{COMPONENTE DE GESTIÓN EDUCATIVA}

En este componente se ha identificado cómo la participación activa de los directivos docentes para el análisis y la contextualización de las estrategias pedagógicas se constituye en un pilar fundamental para alcanzar la calidad educativa, puesto que son estos quienes motivan a los docentes a participar de las formaciones in situ y de las estrategias pedagógicas propuestas. Además, abren las puertas de la institución educativa a la cualificación docente y actualización pedagógica a través de los acuerdos de gestión, la convocatoria de los docentes a participar en las CDA del PTA y a la organización de los recursos necesarios para que se dé la ejecución del programa en la institución educativa. Del mismo modo, los directivos docentes son quienes evalúan qué tanto se han alcanzado los objetivos propuestos en términos de evaluación interna y externa, uso de los materiales educativos y ejecución de la estrategia pedagógica MEC. "Esto implica el desarrollo de pautas y acciones que fortalezcan el liderazgo de los directivos docentes en el mejoramiento del clima escolar y de aula, y de los padres de familia, en la motivación y consolidación de aprendizajes efectivos en sus hijos" (MEN, 2013). Por tal motivo, la implementación del PTA en Puerto Escondido ha motivado a los directivos docentes a ser participantes activos y fundamentales para el reto de alcanzar la calidad educativa en esta región de Córdoba.

\section{COMPONENTE MISIONAL}

Los resultados obtenidos de este componente misional, a través de la implementación del PTA en el municipio de Puerto Escondido - Córdoba, apuntan a que este se construye de forma transversal porque busca la socialización del PTA con cada uno de los actores principales del proceso educativo. Es decir, con la comunidad o padres de familia, organizaciones del estado colombiano, estudiantes, docentes y directivos docentes de la región y la movilización de cada uno, en función de 
los objetivos establecidos en hacer de Colombia la más educada.

Uno de los factores que demuestra este componente misional es el compromiso del municipio de Puerto Escondido en mejorar sus resultados en las pruebas Saber, específicamente en el caso de matemáticas y lenguaje, para lo cual se han generado estrategias de participación en pruebas Supérate con el Saber, simulacros, evaluaciones diagnósticas, todo esto con el aporte significativo de la administración municipal.

Tabla

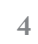

4

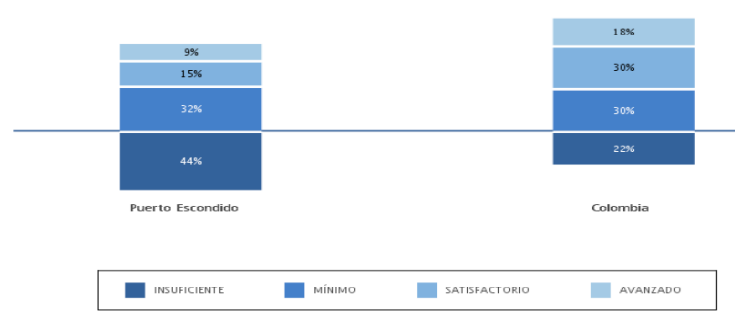

PUERTO ESCONDIDO AÑO 2013

1.1 Comparación de porcentajes según niveles de desempeño en el municipio y el país en matemáticas, tercer grado

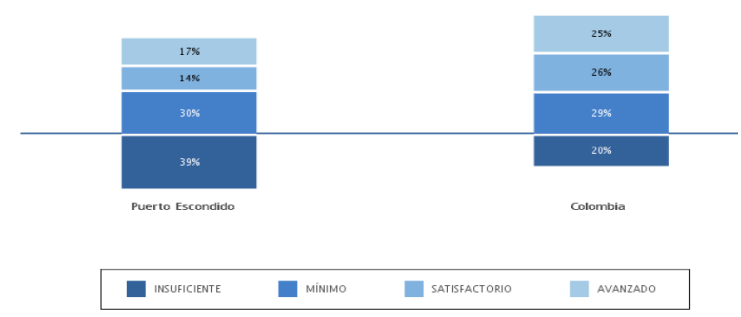

\section{PUERTO ESCONDIDO AÑO 2014}

1.1 Comparación de porcentajes según niveles de desempeño en el municipio y e país en lenguaje, tercer grado

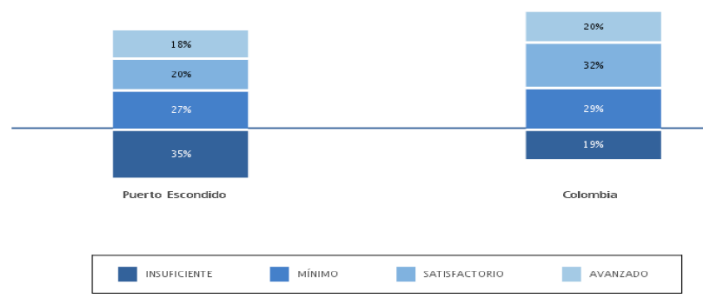

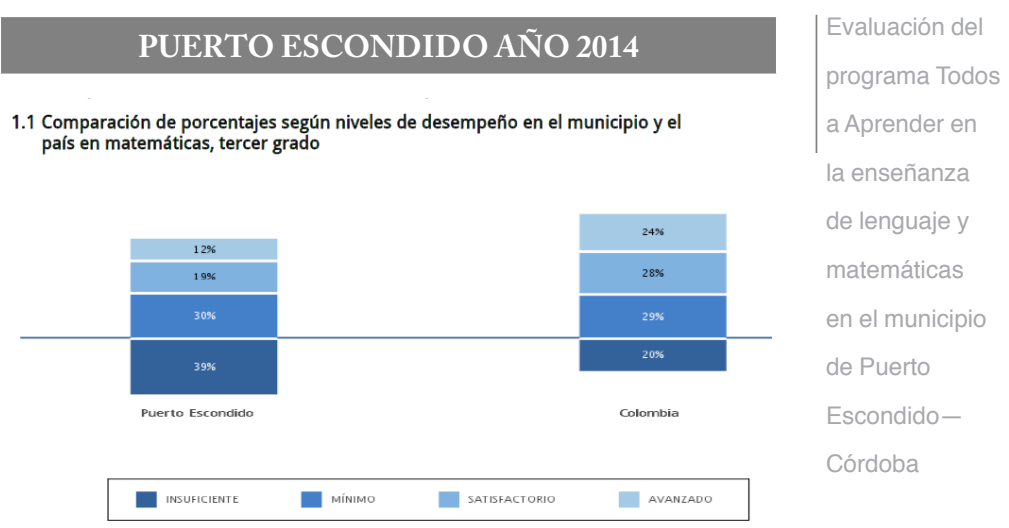

Fuente: Elaboración propia del autor

Se puede evidenciar con estos resultados positivos y de incremento en los niveles de satisfactorio y avanzado para el año 2014, en las áreas de lenguaje y matemáticas, cómo el impacto de las políticas municipales han influido positivamente en el mejoramiento de la calidad educativa de la región de Puerto Escondido. Por tal motivo, "no existe un solo caso en el mundo en el que una institución educativa o un sistema educativo haya podido cambiar drásticamente la trayectoria de los logros de sus estudiantes sin un liderazgo y un compromiso fuerte entre la comunidad educativa y la sociedad en su conjunto" (MEN, 2013). Se puede decir que en esta región se está cumpliendo fielmente con esta situación.

\section{CONCLUSIONES}

Para este proyecto investigativo se derivaron algunas conclusiones, entre las cuales se tiene: las CDA se han convertido en una herramienta de trabajo en equipo para la enseñanza colaborativa y la cualificación docente, permitiendo el mejoramiento continuo, pues los docentes se constituyen en un par académico que evalúa los procesos de enseñanza de ellos mismos a través de la ejecución de la MEC. La participación activa de maestros, directivos docentes, estudiantes y padres de familia fortalecen el proceso de enseñanza aprendizaje, puesto que se crea un espacio de comunicación permanente para el mejoramiento del rendimiento académico del alumno, del acompañamiento del padre de familia y del proceso educativo del docente, todo esto bajo la mirada reflexiva del directivo docente quien funciona como orientador de los procesos académicos, curriculares y administrativos, atendiendo las características básicas
I Panorama I pp. 46-60 I Volumen 10 I Julio-diciembre I 2016 I Número 19 
Alex Mauricio

Díaz | En el uso de materiales educativos para el desarrollo del área de lenguaje y matemáticas, se destaca la motivación constante por parte del estudiante para estar en las sesiones de trabajo de estas dos áreas, lo que es justificado por los docentes como resultado de las actividades de formación situada y el trabajo de las STS, y el acompañamiento en el aula por parte de la CDA y de los procesos de planeación con los docentes integrantes del equipo MEC. Además, el mejoramiento de la instrucción del docente en estas áreas, ya que se utilizan estrategias pedagógicas centradas en el estudiante y bajo las orientaciones pedagógicas de los estándares básicos de competencias, los derechos básicos de aprendizaje y los lineamientos curriculares para la enseñanza de estas dos áreas fundamentales del proceso educativo.

El acompañamiento comprometido de los entes territoriales certificados y de las secretarías de educación contribuyen con el logro de la calidad educativa, pues se constituyen como orientadores del proceso educativo y coordinadores de los lineamientos pedagógicos para cada institución.

\section{REFERENCIAS}

1. Bonilla, C. (2005). Más allá del dilema de los métodos. La investigación en las Ciencias Sociales. Bogotá: Grupo editorial Norma.

2. Castellanos, C. (2012). Los pensamientos de los profesores universitarios. Las concepciones pedagógicas sobre la enseñanza y el aprendizaje en la facultad de ciencias de la salud. Maestría en Educación Sué Caribe. Montería: Universidad de Córdoba.

Panorama I

5. DeWalt, Kathleen M. \& DeWalt, Billie R. (2002). Participant observation: a guide for fieldworkers. Walnut Creek, CA: AltaMira Press.
6. Díaz, S. (2015). Evaluación del programa Todos a Aprender: resultados de la evaluación de contexto. Revista de Estudios e Investigación en Psicología y Educación. España: Universidade da Coruña. (10). 55-59.

7. EXPERIENCIA No. 1: 'Maestro, invítame a tu clase'. El grupo de 'Estudio de Clase' de la Institución Educativa El Hatillo y el desarrollo de clase en la Básica Primaria. Barbosa Antioquia. Año 2007 - 2008. Nivel Institucional. Convenio MEN Colombia - Agencia JICA.

8. EXPERIENCIA No. 2: 'Maestros que aprenden de maestros'. Caso de Bogotá D.C. Año 2007 - 2009. Nivel Regional. Convenio MEN Colombia - Agencia JICA.

9. EXPERIENCIA No. 3: 'Estrategias pedagógicas y metodológicas para la cualificación docente'. Año 2008. Caldas - Nivel Regional. Convenio MEN Colombia - Agencia JICA.

10. EXPERIENCIA No. 4: 'E1 Estudio de Clase y la formación de Licenciados en Matemáticas en la Universidad de Nariño'. Año 2007 - 2008. Nivel Superior. Convenio MEN Colombia Agencia JICA.

11. EXPERIENCIA No. 5: 'Estudio de clase Transformaciones Rígidas'. Cauca - Año 2007. Nivel Regional. Convenio MEN Colombia Agencia JICA.

12. Fernandez, A. (2009). Estudio de Clase. Bogotá: DIGA.

13. Furman, M. (2012). Orientaciones técnicas para la producción de secuencias didácticas para un desarrollo profesional situado en las áreas de matemáticas y ciencias. Bogotá: Ministerio de Educación Nacional.

14. Gobantes, J. (2000). Calidad y evaluación de programas: usos y diseño de la evaluación. En González, T. Evaluación y gestión de la calidad educativa, un enfoque metodológico (p. 83-125). Málaga: Ediciones Aljibe.

15. González, T. (2000). Evaluación y gestión de la calidad educativa. En: Evaluación y gestión de la calidad educativa, un enfoque metodológico (49-79). Málaga: Ediciones Aljibe.

16. Instituto Colombiano para el Fomento de la Educación Superior, ICFES. (2015). Informe resultados nacionales. Saber $3^{\circ}, 5^{\circ}$ y $9^{\circ}$. Bogotá.

17. Martínez, F. (2014). Alternativas para evaluar la calidad social de la educación. Revista iberoamericana de educación. 68(2), 1-14. 
18. McKinsey y Company (2007). How the World's Best-Performing School Systems Come Out On Top. Recuperado de: http://mckinseyonsociety.com/ how-the-worlds-best-performing-schools-come-outon-top/

19. Ministerio de Educación Nacional de Colombia, MEN. (2013). Programa Todos a Aprender para la transformación de la calidad educativa. Guía 1. Sustentos del programa. Recuperado de:

20. http://www.mineducacion.gov.co/1621/ articles31069_archivo_pdf_sustentos_ junio27_2013.pdf

21. Ministerio de Educación Nacional. (2012). Propuesta para la consolidación de los módulos en didáctica del Lenguaje y las matemáticas en el marco del componente de formación situada.

22. Ministerio de Educación Nacional. (2009). Estudio de clase: una experiencia en Colombia para el mejoramiento en las prácticas educativas.

23. Nunan, D. (2003). Collaborative Language Learning and Teaching. Cambridge: Cambridge University Press.

24. OCDE. (2012). Equity and Quality in Education: Supporting Disadvantaged Students and Schools. Recuperado de: http://dx.doi. org/10.1787/9789264130852-en

25. Pérez, R. (2014). Evaluación de programas educativos. Madrid: Editorial la Muralla.

26. Robles, B. (2011). La entrevista en profundidad: una técnica útil dentro del campo antropofísico. Cuicuilco, 18(52), 39-49.

27. Schensul, S. L.; Schensul, J. J. \& LeCompte, M.D. (1999). Essential ethnographic methods: Observations, interviews, and questionnaires (Book 2 en Ethnographer's Toolkit). Walnut Creek, CA: AltaMira Press.

28. Taylor, S. J. \& Bogdan, R. (1987). Introducción a los métodos cualitativos de investigación. La búsqueda de significados. Barcelona: Paidos.

29. UNESCO (2004). Educación para todos, el imperativo de la calidad. Informe de seguimiento de la EPT en el mundo. París: Graphoprint.

30. UNESCO (2013). Situación Educativa de América Latina y el Caribe: hacia la Educación de Calidad para todos al 2015. Santiago: Oficina Regional de Educación para América Latina y el Caribe.

31. Valls, R. (2005). Revista de educación social. Recuperado de: http://www.eduso.net/ $\mathrm{res} / \mathrm{?b}=7 \& \mathrm{c}=53 \& \mathrm{z}=145$
32. Vezub, L. (2009). Organización de las Naciones Unidas para la Educación, la Ciencia y la Cultura (Unesco). Recuperado de: http://www.iipe-buenosaires.org.ar
Evaluación del
programa Todos
a Aprender en
la enseñanza

de lenguaje y

matemáticas

en el municipio

de Puerto

Escondido-

Córdoba 